\title{
Análise da reprodutibilidade de parâmetros no domínio da frequência do sinal EMG utilizados na caracterização da fadiga muscular localizada
}

\author{
Cristiano Rocha da Silva ${ }^{1}$ \\ Beatriz Sanches Geres ${ }^{1}$ \\ Heloyse Uliam Kuriki ${ }^{1,2}$

Resumo: O objetivo deste estudo foi analisar a reprodutibilidade de parâmetros no domínio da frequência do sinal eletromiográfico (EMG) utilizados na caracterização da fadiga muscular localizada. Quinze sujeitos do sexo masculino foram submetidos a um teste de fadiga baseado na extensão isométrica de joelho, sendo realizados em três momentos distintos com intervalos de sete dias. Para avaliar a reprodutibilidade dos dados entres os testes calculou-se o coeficiente de correlação intraclasse $(\mathrm{CCl})$ para a frequência mediana (Fmed) no tempo total de exercício $\left(\right.$ Fmed $\left._{T}\right)$, para a Fmed obtida a cada $10 \%$ do tempo de exercício $\left(\mathrm{Fmed}_{10 \%}\right)$ e para as potências das bandas de frequência, obtidas da divisão do espectro de potência a cada $20 \mathrm{~Hz}$. Os resultados demonstraram: (1) boa reprodutibilidade para a Fmed $\mathrm{T}_{\mathrm{T}}$; (2) boa reprodutibilidade para a Fmed $_{10 \%}$; e (3) maior variação no sinal EMG nas bandas de $20 \mathrm{a} 120 \mathrm{~Hz}$, no qual se destacam as bandas de $20-40 \mathrm{~Hz}$ e de $40-60 \mathrm{~Hz}$, demonstrando maior sensibilidade ao processo de fadiga muscular. Conclui-se que a Fmed é uma variável que apresenta boa reprodutibilidade e que a análise fragmentada do espectro de potência, por meio das bandas de frequência, demonstrou-se sensível as variações que ocorrem no sinal EMG durante a instalação do processo de fadiga, tendo potencial para se tornar um novo método para a caracterização da fadiga muscular localizada.

Palavras-chave: Eletromiografia. Fadiga. Reprodutibilidade dos testes. Biomecânica. Músculo quadríceps. Frequência mediana.

\section{Analysis of reliability of EMG signal frequency domain parameters used in the characterization of localized muscle fatigue}

\begin{abstract}
The aim of this study was to analyze the reproducibility of the electromyography signal's parameters (EMG) in the frequency domain used in the characterization of localized muscle fatigue. Fifteen male subjects underwent a fatigue test based on isometric knee extension, being held at three different times at intervals of seven days. To assess the reproducibility of data between the tests we calculated the correlation coefficient (ICC) for the median frequency (MF) in total exercise time $\left(\mathrm{MF}_{\mathrm{T}}\right)$, MF obtained for every $10 \%$ of exercise time $\left(\mathrm{MF}_{10 \%}\right)$ and the powers of the frequency bands obtained by dividing the power spectrum at windows of $20 \mathrm{~Hz}$. The results showed: (1) excellent reproducibility for $\mathrm{MF}_{\mathrm{T}}$, (2) good reproducibility for $\mathrm{MF}_{10 \%}$, and (3) greater variation in the signal EMG bands from 20 to $120 \mathrm{~Hz}$, especially at the bands of $20-40 \mathrm{~Hz}$ and $40-60 \mathrm{~Hz}$, which showed greater sensitivity to the process of muscle fatigue. We conclude that the MF is a variable that shows good reproducibility and that the fragmented analysis of the power spectrum, by means of frequency bands, showed that significant variations occur in the EMG signal during the installation of the fatigue process, having potential to become a new method for the characterization of localized muscle fatigue.
\end{abstract}

Keywords: Electromyography. Fatigue. Reproducibility of results. Biomechanics. Quadriceps muscle. Median frequency.

\section{Introdução}

A fadiga muscular pode ser definida como qualquer redução na capacidade do sistema neuromuscular em gerar força (ZWARTS et al.,
2008). A perda da capacidade do músculo em gerar força pode ter duas origens: uma central e uma periférica. A fadiga de origem central pode ser definida como uma degradação progressiva 
da ativação muscular induzida pelo exercício, abrangendo todos os fenômenos fisiológicos espinais e supraespinais capazes de diminuir a excitação dos motoneurônios. Já na fadiga muscular periférica ocorrem alterações na propagação do potencial de ação muscular, nos mecanismos contráteis do músculo e na concentração de íons importantes para a manutenção da contração muscular (BOYAS; GUÉVEL, 2011). Baron et al. (2008) propõem que a fadiga é um processo fisiológico mais complexo que não estaria relacionado à falência de qualquer sistema fisiológico, mas sim, estes seriam sinalizadores ao sistema nervoso central (SNC), a fim de evitar danos irreversíveis ou até mesmo morte celular.

O entendimento das causas da fadiga muscular constitui uma área temática que, apesar de ser bastante estudada, ainda apresenta controvérsia. Por ser considerada um conceito multidimensional que abrange aspectos fisiológicos e psicológicos, isolar e quantificar os sintomas que precedem a perda na capacidade do músculo em sustentar um nível de força, caracteriza um problema de elevada complexidade no meio clínico e esportivo (ZWARTS et al., 2008).

A biomecânica é uma área da ciência que vem contribuindo na integração de conceitos, ferramentas e métodos objetivando um entendimento mais amplo da fadiga, permitindo a operação de análises dos padrões dos impulsos neuromusculares durante o exercício por meio da eletromiografia de superfície. Sabe-se que a fadiga muscular é um processo que gera alterações nos padrões de ativação muscular, tendo a análise do sinal eletromiográfico (EMG) importante papel na sua avaliação (CIFREK et al., 2009; DIDERIKSEN et al., 2010). Desta maneira, entende-se que ela pode ser usada como uma ferramenta que permite avaliar as alterações decorrentes da fadiga muscular, tendo em vista que os processos fisiológicos que reduzem a capacidade do músculo em gerar força provavelmente começam antes que esse declínio possa ser observado (ROGERS; MACISAAC, 2010).

Usualmente, tem-se utilizado a análise no domínio da frequência do sinal EMG para a caracterização do processo de fadiga muscular localizada (ALLISON; FUJIWARA, 2002; SANTOS et al., 2008; OLIVEIRA; GONÇALVES, 2009). Estudos demonstram haver diminuição nos valores da Fmed durante o processo de instalação da fadiga muscular localizada, sendo um dos indicadores utilizados em métodos para a quantificação deste processo (GONZALEZ-IZAL, et. al. 2010). Allison e Fujiwara (2002) relatam que mudanças relativas na amplitude de frequência de bandas específicas, apresentam alta correlação com a variação da Fmed. Porém, em alguns casos, a alteração em uma determinada banda do espectro pode ser compensada por alteração em outras bandas, não resultando em uma mudança efetiva no valor da Fmed (DE LUCA, 1997). Sendo assim, pequenas alterações nos componentes de frequência do espectro poderiam, em hipótese, não determinar alterações na Fmed. Nesse sentido pode-se inferir que determinadas bandas de frequência podem apresentar uma sensibilidade maior aos fatores fisiológicos que determinam a instalação do processo de fadiga muscular. Consequentemente, a análise isolada destas bandas pode representar uma forma mais precisa de monitoração do processo de fadiga muscular. (ALLISON E FUJIWARA, 2002;

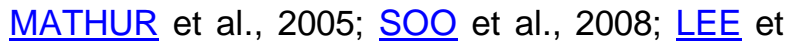
al., 2009; $\underline{\mathrm{SOO}}$ et al., 2010).

Diante do exposto, entende-se que a variação da Fmed pode interferir na sua representatividade em relação ao fenômeno da fadiga muscular localizada. Apesar da variação, entende-se que a Fmed pode ser semelhante entre testes, na qual uma alta correlação entre os testes pode atestar que ela é reprodutível, porém variável. Neste contexto, o objetivo do presente estudo reside na análise da reprodutibilidade da Fmed por meio da observação do comportamento da densidade espectral do sinal EMG e de suas bandas isoladas, utilizados na caracterização da fadiga muscular localizada.

\section{Materiais e Métodos}

\section{Grupo amostral}

Foram selecionados 15 sujeitos, do sexo masculino, com idade de 21,93 $\pm 2,40$ anos, massa de $80,26 \pm 8,88 \mathrm{~kg}$ e altura de 177,4 \pm $2,58 \mathrm{~cm}$. A amostra foi composta de sujeitos que não apresentassem qualquer desordem neuromuscular e/ou articular, no membro inferior dominante, em um período inferior a 18 meses do momento de coleta de dados. O membro dominante foi determinado baseando-se na preferência do sujeito em realizar um gesto de chute (EBERSOLE et al., 2006). Antes da realização dos testes, todos os sujeitos selecionados visitaram o laboratório e foram orientados sobre sua participação no estudo e procedimentos adotados. Após os esclarecimentos, assinaram um termo de consentimento livre e esclarecido. Os 
procedimentos utilizados neste estudo foram analisados pela comissão de ética local, da FCTUNESP Campus de Presidente Prudente, obtendo parecer favorável por meio do processo no 17/2009.

\section{Protocolo de Avaliação}

Inicialmente o sujeito foi posicionado sentado em uma mesa extensora de joelho (marca VITTALY ${ }^{\circledR}$, modelo convergent ${ }^{\circledR}$ ), com ajustes do apoio para as costas e do comprimento da alavanca de resistência para a perna do membro inferior dominante. O ângulo da articulação do joelho nesta alavanca foi limitado a $60^{\circ}$, para favorecer a maior geração de força por parte do quadríceps (PINCIVERO, 2003).

Com o objetivo de induzir à fadiga dos músculos reto femoral (RF), vasto lateral (VL) e vasto medial (VM) do quadríceps femoral, os sujeitos realizaram: i) teste máximo por meio da Contração Voluntária Isométrica Máxima (CVIM), para posteriormente determinar um nível de esforço submáximo, ii) teste de fadiga, que consistiu da sustentação da extensão do joelho em um nível de $50 \%$ da CVIM até a exaustão.

Para determinar a CVIM, o sujeito realizou três contrações sustentadas por 6 segundos com um descanso de 10 minutos entre elas. Dos 6 segundos foram desconsiderados da análise os dois primeiros e dois últimos. Assim, foi considerada como CVIM a média geral dos dados obtidos neste intervalo para as três contrações.

Após sete dias da realização do teste de CVIM, o sujeito realizou o primeiro teste de fadiga, no qual sustentou a extensão de joelho com intensidade de carga igual a $50 \%$ da CVIM. $\mathrm{O}$ sujeito foi orientado a sustentar a carga até a exaustão. Considerou-se como exaustão o momento em que ele cessasse completamente 0 exercício ou apresentasse incapacidade na manutenção da carga, pré-determinada, representada pela queda de $10 \%$ na sua intensidade. Para auxiliar no controle da intensidade da carga, a força aplicada foi monitorada por meio de uma célula de carga (modelo MM - $100 \mathrm{Kgf}$, marca $\mathrm{Kratos}^{\circledR}$, São Paulo-SP, Brasil), representando um feedback visual em tempo real. Este feedback foi fornecido por meio de um monitor de computador e do programa de aquisição de dados, permitindo a manutenção do nível de esforço pelo sujeito. $O$ teste de fadiga foi realizado em três momentos com intervalos de sete dias.

\section{Aquisição dos sinais}

Para a aquisição do sinal EMG foram utilizados três pares de eletrodos de superfície, em configuração bipolar (modelo Meditrace ${ }^{\circledR}$ da marca $3 \mathrm{M}^{\circledast}$, com superfícies de captação de $\mathrm{Ag} / \mathrm{AgCl}$ com $10 \mathrm{~mm}$ de diâmetro). O eletrodo de referência foi posicionado no processo estiloide da Ulna. No cabo do eletrodo estava presente um circuito pré-amplificador com ganho de 20 vezes, Modo de Rejeição Comum maior que $80 \mathrm{~dB}$ e impedância de $1 \mathrm{~K} \Omega$. Por meio de um aparelho de eletroestimulação modelo Nemesys 942 (marca Quark ${ }^{\circledR}$ ) e um eletrodo tipo "caneta", foram localizados os pontos motores das porções VM, VL e RF do músculo quadríceps femoral. Após a localização e marcação de cada ponto motor, foi realizada a tricotomia e a limpeza da região. Os eletrodos foram posicionados paralelamente, separados entre si por $20 \mathrm{~mm}$ e fixados a aproximadamente $2 \mathrm{~cm}$ do ponto motor na direção da fibras musculares sobre cada um dos ventres musculares (DE LUCA, 1997; MATHUR et al., 2005; EBERSOLE et al., 2006; AZEVEDO, 2007).

Com o objetivo de garantir o mesmo posicionamento dos eletrodos nos três dias de coleta de dados, foi desenvolvido um sistema de gabarito que consistiu de uma folha de acetato, no qual se demarcava a borda superior da patela, a área tricotomizada de cada uma das porções musculares e o ponto motor. Quando o sujeito retornou para novo dia de teste, essa transparência foi alinhada com a borda superior da patela e as áreas de tricotomia, e a partir disso, a marcação dos pontos foi realizada.

Os sinais foram captados em um módulo condicionador de sinais (modelo BIO EMG 10008-4I, marca LYNX ${ }^{\circledR}$ Tecnologia Eletrônica Ltda, São Paulo-SP, Brasil). Neste módulo três canais para a aquisição de sinais EMG foram configurados com um filtro digital, passa-baixa com frequência de corte de $500 \mathrm{~Hz}$ e um passaalta com frequência de corte de $20 \mathrm{~Hz}$. Todos os canais apresentaram ganho final de 1000 vezes e frequência de amostragem de $4000 \mathrm{~Hz}$. A aquisição e o armazenamento dos sinais em arquivos de dados foram feitos através do software Bioinspector $1.8\left(\mathrm{LYNX}^{\circledR}\right)$. Durante o teste de fadiga a monitoração da força aplicada pelo sujeito foi realizada por meio de uma célula de carga sincronizada com a coleta dos sinais EMG, tendo no condicionador de sinais um canal configurado para a aquisição de seus sinais, habilitando-se um filtro digital passa baixa de 100 $\mathrm{Hz}$.

\section{Processamento dos sinais}

Os sinais de EMG coletados durante os testes de fadiga foram processados por meio de um 
algoritmo desenvolvido em ambiente MatLab ${ }^{\circledR}$. Todos os procedimentos descritos foram executados para os sinais coletados nos três momentos distintos.

Foram desconsiderados da análise os dois primeiros e dois últimos segundos do sinal EMG provenientes do teste de fadiga. Aplicou-se ao sinal EMG bruto, um filtro digital passa-banda tipo Butterworth de $4^{\mathrm{a}}$ ordem e frequência de corte entre 20 e $500 \mathrm{~Hz}$. Os dados fora submetidos à Transformada Discreta de Fourier (TDF), com janela de $1000 \mathrm{~ms}$ e overlap de 500 ms, para o cálculo de seu espectro de potência. O espectro de potência calculado foi normalizado por meio da derivação de sua função de distribuição espectral. A partir do espectro de potência foi calculada a Fmed, considerada como a frequência que divide o espectro de potência do sinal em duas áreas iguais. Ela foi normalizada pela Fmed inicial $\left(\mathrm{Fmed}_{1}\right)$. A Fmed $\mathrm{f}_{1}$ foi obtida por meio da extração das cinco primeiras amostras de Fmed do espectro de potência. Deste modo, considerou-se como sendo o início a média das amostras de Fmed tiradas do início do exercício e que representavam o sujeito "descansado". Diante disso, todos os valores de Fmed durante os testes foram divididos pela Fmed, tendo uma queda percentual com o aparecimento da fadiga. Para a análise das bandas de frequência, 0 espectro normalizado foi dividido na faixa de 20 $400 \mathrm{~Hz}$ com intervalos de $20 \mathrm{~Hz}$.

\section{Tratamento estatístico}

A reprodutibilidade das variáveis em estudo foi analisada por meio do Coeficiente de Correlação Intraclasse $(\mathrm{CCl})$. O $\mathrm{CCl}$ utilizado no presente estudo é o modelo $\mathrm{CCl}_{2,1}$ (modelo two way random com concordância absoluta). $\mathrm{O} \mathrm{CCl}$ é um índice que varia de 0,00 até 1,00, onde valores entre 0,80-1,00 representam excelente reprodutibilidade, de 0,60-0,80 indicam boa reprodutibilidade, enquanto $\mathrm{CCl}$ abaixo de 0,60 reflete uma reprodutibilidade pobre (BARTKO, 1996; PORTNEY; WATKINS, 2009). No presente estudo o $\mathrm{CCl}$ foi calculado para os valores de Fmed no tempo total de exercício $\left(\mathrm{Fmed}_{\mathrm{T}}\right)$, para a Fmed obtida a cada $10 \%$ do tempo de exercício $\left(\right.$ Fmed $\left._{10 \%}\right)$ e para as potências de cada uma das bandas de frequência.

A normalidade dos dados foi verificada por meio do teste de Shapiro-Wilk. As variáveis analisadas foram a Fmed final $\left(\right.$ Fmed $\left._{F}\right)$ do VM, RF e VL obtidas nos três dias de testes. Para o tratamento dos dados foi aplicada uma ANOVA de dois fatores para dados repetidos (músculos $X$ dias) e para as comparações entre fatores foi utilizado o teste Post Hoc de Tukey. Para verificar a instalação do processo de fadiga, foi aplicado um teste $t$ (one-sample) aos valores de $\mathrm{Fmed}_{\mathrm{F}}$ do VM, RF e VL. Na análise descritiva dos dados, além das médias e dos desvios padrões, também são apresentados os coeficientes de variação $(\mathrm{CV})$, representando em porcentagem o quanto que as variáveis se dispersaram em torno da média. As análises foram realizadas utilizando-se o pacote estatístico SPSS 18.0 para Windows. Para todos os testes, foi considerado um nível de significância de $5 \%$.

\section{Resultados}

1. Frequência Mediana. Os valores observados na Tabela 1 demonstram que 0 protocolo executado no estudo induziu os músculos avaliados ao processo de fadiga muscular localizada $(p<0,05)$. Cabe lembrar que os valores de Fmed foram normalizados pela Fmed, dessa maneira, possuíam no início de cada teste valores iguais a um. Sabe-se que em um protocolo de fadiga muscular localizada há uma diminuição dos valores de Fmed (YASSIERLI; NUSSBAUM, 2008), conforme pode ser observado na Tabela 1. Foram realizadas comparações entre os dias para um mesmo músculo, porém não foram encontradas diferenças significantes no VM, VL e RF.

Nos testes máximos os valores das CVIMs foram de 476,40 $\pm 149,15 \mathrm{~N}$ e durante os testes de fadiga a $50 \%$ da CVIM foram de $234,57 \pm$ $81,78 \mathrm{~N}$.

Tabela 1. Média e desvio padrão dos valores

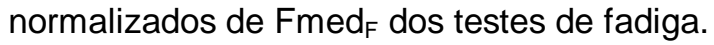

\begin{tabular}{cccc}
\hline Músculos & Momento 1 & Momento 2 & Momento 3 \\
\hline$V M$ & $0,89 \pm 0,08$ & $0,85 \pm 0,11$ & $0,91 \pm 0,14$ \\
$V L$ & $0,86 \pm 0,06$ & $0,83 \pm 0,11$ & $0,85 \pm 0,07$ \\
$R F$ & $0,76 \pm 0,09$ & $0,79 \pm 0,09$ & $0,82 \pm 0,09$ \\
\hline
\end{tabular}

2. Análise do $\mathrm{CCl}$ da Fmed F $_{\text {. Ao considerar os }}$ valores de Fmed obtidos durante o tempo total de exercício para as três porções musculares, o $\mathrm{CCl}$ apresentou excelente correlação para o VM $(0,93$ $[0,89-0,98]), \operatorname{VL}(0,93[0,88-0,98])$ e o $\operatorname{RF}(0,88$ $[0,78$ - 0,98]), indicando uma excelente reprodutibilidade dos dados ao longo das três sessões de teste. Tecnicamente a porção do RF apresentou excelente correlação, no entanto, na análise observa-se que este apresenta um maior CV $(19,3 \%)$ quando comparado com as porções do VM $(8,6 \%)$ e VL $(8,6 \%)$.

3. Análise do $\mathrm{CCl}$ da Fmed $_{10 \%}$. Pode-se aprofundar 0 entendimento dos resultados 
apresentados quando os valores da Fmed são fragmentados de 10 em $10 \%$ do tempo total do teste de fadiga. Observa-se que para o VM e VL, os valores de $\mathrm{CCl}$ encontram-se entre $0,77 \mathrm{e}$ 0,89 , e para o RF, têm-se valores entre 0,70 e 0,87 . Pode-se inferir a existência de um padrão na reprodutibilidade da Fmed para as três porções, no qual ocorre: i) um momento inicial I que apresenta ligeira queda do $\mathrm{CCl}$; ii) um momento II que apresenta recuperação (aumento) do $\mathrm{CCl}$; iii) um momento III que apresenta uma ligeira queda do $\mathrm{CCl}$. Nota-se também que os valores máximos de $\mathrm{CCl}$ ocorreram em $60 \%$ do tempo total de exercício para o VM e VL e em $50 \%$ para o RF. A Tabela 2 apresenta os valores de $\mathrm{CCl}$ calculados para as três porções musculares.

Tabela 2. Valores de CCl e seus respectivos intervalos de confiança $(95 \%)$ para a Fmed a cada $10 \%$ do tempo de exercício (TE), para os músculos VM, VL e RF nos três testes de fadiga.

\begin{tabular}{|c|c|c|c|c|}
\hline Momento & $\begin{array}{c}\% \\
\text { TE }\end{array}$ & VM & VL & RF \\
\hline \multirow[b]{2}{*}{ I } & 10 & $0,86(0,73-0,99)$ & $0,81(0,68-0,92)$ & $0,85(0,76-0,94)$ \\
\hline & 20 & $0,79(0,65-0,92)$ & $0,79(0,65-0,92)$ & $0,74(0,55-0,91)$ \\
\hline \multirow{4}{*}{ II } & 30 & $0,80(0,63-0,95)$ & $0,82(0,67-0,95)$ & $0,82(0,67-0,95)$ \\
\hline & 40 & $0,82(0,69-0,93)$ & $0,77(0,61-0,93)$ & $0,78(0,62-0,94)$ \\
\hline & 50 & $0,86(0,77-0,95)$ & $0,80(0,65-0,94)$ & $0,87(0,82-0,91)$ \\
\hline & 60 & $0,88(0,80-0,95)$ & $0,89(0,84-0,93)$ & $0,72(0,55-0,87)$ \\
\hline \multirow{4}{*}{ III } & 70 & $0,79(0,66-0,92)$ & $0,85(0,74-0,94)$ & $0,71(0,58-0,83)$ \\
\hline & 80 & $0,87(0,76-0,97)$ & $0,87(0,79-0,95)$ & $0,81(0,69-0,92)$ \\
\hline & 90 & $0,87(0,75-0,97)$ & $0,80(0,64-0,95)$ & $0,70(0,55-0,84)$ \\
\hline & 100 & $0,78(0,63-0,92)$ & $0,85(0,75-0,94)$ & $0,71(0,55-0,86)$ \\
\hline
\end{tabular}

Para todos os valores de CCI calculados, o F-Test foi significante $(<0,05)$.

4. Análise do $\mathrm{CCl}$ das bandas de frequência isoladas do sinal EMG. Quando analisadas as potências das bandas de frequência, referentes ao tempo total dos testes de fadiga notam-se valores de $\mathrm{CCl}$ qualificados entre bom e excelente. As faixas abaixo de $120 \mathrm{~Hz}$ foram as que apresentaram menor reprodutibilidade dentro do conjunto, e dentre elas, as faixas de $20-40 \mathrm{~Hz}$ e de $40-60 \mathrm{~Hz}$ são as que apresentam menor $\mathrm{CCl}$ e maior CV, como podem ser observadas na Tabela 3, que traz os valores médios de $\mathrm{CCl}$ e o CV para as três porções musculares.

Tabela 3. Média $(\overline{\mathrm{X}})$, intervalos de confiança (IC - 95\%) e coeficiente de variação (CV) de CCI das bandas de frequência selecionadas para análise, das três porções musculares, considerando o tempo total de exercício dos três testes de fadiga.

\begin{tabular}{ccccccc}
\hline \multirow{2}{*}{ Bandas } & \multicolumn{1}{c}{ VM } & \multicolumn{2}{c}{ VL } & \multicolumn{2}{c}{ RF } \\
\cline { 2 - 7 } & $\overline{\mathbf{x}}$ e IC $-95 \%$ & $\mathbf{C V}$ & $\overline{\mathbf{x}}$ e IC $-95 \%$ & $\mathbf{C V}$ & $\overline{\mathbf{x}}$ e IC $-95 \%$ & $\mathbf{c V}$ \\
\hline $20-40 \mathrm{hz}$ & $0,90(0,77-1,00)$ & 24,4 & $0,83(0,69-0,97)$ & 30,1 & $0,84(0,75-0,94)$ & 20,2 \\
\hline $40-60 \mathrm{hz}$ & $0,92(0,83-0,99)$ & 16,3 & $0,85(0,74-1,00)$ & 27,0 & $0,81(0,67-0,96)$ & 32,0 \\
\hline $60-80 \mathrm{hz}$ & $0,89(0,84-0,93)$ & 8,9 & $0,89(0,81-0,96)$ & 14,6 & $0,89(0,84-0,94)$ & 8,9 \\
\hline $80-100 \mathrm{hz}$ & $0,95(0,91-0,99)$ & 7,3 & $0,95(0,92-0,98)$ & 5,2 & $0,83(0,70-0,96)$ & 25,8 \\
\hline $100-120 \mathrm{hz}$ & $0,95(0,91-1,00)$ & 8,4 & $0,94(0,89-0,98)$ & 8,5 & $0,90(0,85-0,95)$ & 10,0 \\
\hline $120-140 \mathrm{hz}$ & $0,97(0,95-0,99)$ & 3,0 & $0,96(0,91-0,99)$ & 8,3 & $0,85(0,74-0,89)$ & 22,3 \\
\hline
\end{tabular}

Para todos os valores de $\mathrm{CCl}$ calculados, o F-Test foi significante $(<0,05)$. 


\section{Discussão}

O propósito deste estudo foi analisar a reprodutibilidade da Fmed utilizada na caracterização da fadiga muscular localizada por meio da observação do comportamento da densidade espectral do sinal EMG, além de verificar a reprodutibilidade da potência de bandas de frequência, no intuito de identificar as bandas que apresentam maior sensibilidade ao processo de fadiga muscular localizada. Os resultados demonstram que a queda apresentada pela $\mathrm{Fmed}_{\mathrm{T}}$ durante os testes foi reprodutível, reforçando entendimento de que a queda da Fmed em função do tempo de exercício pode ser considerada um indicador global e reprodutível para a caracterização do processo de fadiga muscular localizada (KELLIS; KATIS, 2008; LARIVIÈRE et al., 2008; CALLAGHAN et al., 2009).

Apesar da boa reprodutibilidade da Fmed para as três porções musculares, o RF destoa do VM e $\mathrm{VL}$, apresentando um menor $\mathrm{CCl}$ e um maior $\mathrm{CV}$. Esse comportamento pode ser justificado por uma possível diferença morfológica ( et al., 1973) e biomecânica (JACOBS; VAN INGEN SCHENAU, 1992) entre os músculos do quadríceps. Sabe-se que o RF apresenta maior proporção de fibras do tipo II em relação ao VM e $\mathrm{VL}$, o que o torna mais suscetível à fadiga (JOHNSON et al., 1973; KOUZAKI et al., 1999). No que diz respeito à biomecânica, o VM e VL são músculos monoarticulares e possuem alta capacidade de produção de força. Por outro lado, o RF se constitui em um músculo biarticular, tendo função de distribuir o torque para ambas articulações e controlar a direção do movimento (JACOBS; VAN INGEN SCHENAU, 1992), o que sugere um possível estratégia de controle neural diferenciada daquela dos músculos monoarticulares (KOUZAKI et al., 1999).

Diante das informações apresentadas acima, pode-se inferir que o comportamento da reprodutibilidade de um músculo pode estar relacionado com a sua capacidade de resistir à fadiga, onde os músculos com maior proporção de fibras tipo II tendem a ter menores valores de $\mathrm{CCl}$ devido à sua característica de baixa resistência ao exercício, entretanto o presente estudo não avaliou diretamente a morfologia ou as estratégias de controle neural dos músculos VM, VL e RF, sendo necessária a realização de mais estudos para que estas e outras questões possam ser compreendidas de uma melhor forma, aprofundando o entendimento sobre os fatores mecânicos e fisiológicos que podem influenciar o comportamento do sinal EMG durante a realização de contrações submáximas sustentadas.

Quanto à análise da $\mathrm{Fmed}_{10 \%}$, nota-se que, para o VM e VL, os valores médios do $\mathrm{CCl}$ ficaram entre 0,77 e 0,89, e para o RF, entre 0,70 e 0,87 . Entende-se que, quando o tempo de exercício é fragmentado, é possível observar as variações presentes em cada momento do teste, permitindo analisar quais momentos apresentam maior variação no comportamento da Fmed. $\mathrm{Na}$ análise da $\mathrm{Fmed}_{10 \%}$, pode-se inferir a existência de um padrão em sua reprodutibilidade, ocorrendo nas três porções musculares: i) um momento inicial (momento I), que apresenta ligeiro declínio do $\mathrm{CCl}$; ii) um momento intermediário que apresenta recuperação (aumento) do CCl (momento II); iii) um momento final que apresenta uma ligeira queda do $\mathrm{CCl}$ (momento III) (Tabela 2).

Azevedo (2007) monitorou o comportamento da Fmed em sujeitos que realizaram teste de fadiga com o objetivo de determinar o índice de fadiga para as porções do VM, VL e RF. O autor calculou o coeficiente de inclinação da Fmed e considerou como início de fadiga muscular o momento quando esta inclinação apresentasse valor significativo diferente de zero. Os resultados desse estudo mostraram que 0 momento de fadiga para o VM ocorre em $57 \pm 4 \%$, para o VL em $58 \pm 7 \%$ e para o RF em $47 \pm 8 \%$ do tempo total de exercício. Em nosso estudo, os valores máximos de $\mathrm{CCl}$ ocorreram em $60 \%$ do tempo total de exercício para VM e VL e $50 \%$ para a porção do RF. Comparando o presente estudo com os achados de Azevedo (2007), entende-se que o pico do $\mathrm{CCl}$, assim como o momento de inflexão da reta através da análise do coeficiente de inclinação, pode representar o momento no qual ocorre a instalação do processo de fadiga muscular.

Observa-se que a variação da Fmed no início do momento II é alta e gradativamente aumenta até atingir o valor máximo, ou seja, a reprodutibilidade menor no início pode ser o indicativo de que nesta situação cada sujeito apresenta um comportamento único, porém na transição para o trabalho em regime de fadiga, 
esse comportamento tende a se reproduzir melhor inter-sujeitos.

Outro achado importante dentro da análise da reprodutibilidade da Fmed está relacionado ao comportamento do músculo VL. Observa-se, no final do momento III, que o valor de $\mathrm{CCl}$ tende a ser próximo, sem diferença significante, do máximo que ocorre em $60 \%$ do tempo de exercício. Azevedo (2007) também demonstrou evidências de que, quando o sujeito chega à condição de exaustão, os valores de Fmed para o $\mathrm{VL}$ obtidos nesse momento não apresentam diferenças inter-sujeitos.

Calculou-se também o $\mathrm{CCl}$ para as potências das bandas de frequência, divididas de 20 em 20 $\mathrm{Hz}$ do espectro de potência do sinal EMG. Foram consideradas para análise somente as bandas abaixo de $120 \mathrm{~Hz}$, pois se observou que, quanto maior a frequência das bandas, maior o $\mathrm{CCl}$ e menor o CV, não havendo mudanças em suas potências durante o teste. Uma justificativa para este comportamento está no fato de que nessas faixas, acima de $120 \mathrm{~Hz}$, a quantidade de sinal existente é muito pequena e vai diminuindo com o aumento das bandas de frequência, elevando os valores de $\mathrm{CCl}$. Em contrapartida, ocorreu maior variação nas bandas de baixa frequência, pois a concentração do sinal apresentou tendência a ser maior nessas faixas de frequência. Sabe-se que existe uma tendência de ocorrer um incremento nestas bandas, com uma consequente diminuição das bandas de alta frequência (LOWERY et al., 2002).

A compressão do espectro para componentes de baixa frequência caracteriza um indicador de que o músculo exercitado está trabalhando em regime de fadiga (DE LUCA, 1997; KUMAR, 2006). Estudos anteriores relatam uma significante correlação entre a amplitude das bandas de baixa frequência e a fadiga muscular ( $\underline{\mathrm{SOO}}$ et al., 2008). Em nosso estudo, observouse que essas faixas foram as que apresentaram um menor $\mathrm{CCl}$, possibilitando o entendimento de que as faixas com menor reprodutibilidade podem ser as que demonstram ter maior sensibilidade ao processo de fadiga muscular.

\section{Conclusões}

Os resultados apresentados indicam que 0 comportamento da Fmed $\mathrm{T}$ e da $\mathrm{Fmed}_{10 \%}$ demonstram boa reprodutibilidade na caracterização do processo de fadiga muscular. A observação fragmentada do espectro de potência do sinal EMG revelou ter um potencial maior para a seleção de uma variável que seja mais sensível quando comparada com a Fmed. As faixas abaixo de $120 \mathrm{~Hz}$ foram as que apresentaram menor reprodutibilidade dentro do conjunto de bandas, e dentre elas, as bandas de $20-40 \mathrm{~Hz}$ e de $40-60 \mathrm{~Hz}$ demonstraram maior sensibilidade às variações promovidas pelo processo de fadiga muscular localizada. Ressalta-se que este apontamento é especulativo, porém acredita-se que estas observações apresentam um potencial na contribuição para o desenvolvimento de métodos alternativos mais eficientes e práticos para a caracterização da fadiga muscular localizada.

\section{Referências}

ALLISON, G.T.; FUJIWARA, T. The relationship between EMG median frequency and low frequency band amplitude changes at different levels of muscle capacity. Clinical

Biomechanics, Huddersfield, v. 17, n. 6, p. 4649, 2002. doi: http://dx.doi.org/10.1016/S02680033(02)00033-5

\section{AZEVEDO, F.M. Avaliação do sinal eletromiográfico como parâmetro para a determinação do limiar de fadiga muscular. 2007. 115 f. Tese (Doutorado em Educação Física) - EEFE, Universidade de São Paulo, São Paulo, 2007. Disponível em: \\ <http://www.teses.usp.br/teses/disponiveis/39/391 32/tde-08082008-163407/>. Acesso em: 10 jan. 2011.}

BARON, B. et al. Why does exercise terminate at the maximal lactate steady state intensity? British Journal of Sports Medicine, Londres, v. 42, n. 10 , p. 828-833, 2008. doi: http://dx.doi.org/10.1136/bjsm.2007.040444

BARTKO, J. J. The intraclass correlation coefficient as a measure of reliability.

Psychological Reports, Missoula, v. 19, n. 1, p. 3-11, 1966. doi: http://dx.doi.org/10.2466/pr0.1966.19.1.3

BOYAS, S.; GUÉVEL, A. Neuromuscular fatigue in healthy muscle: Underlying factors and adaptation mechanisms. Annals of Physical and Rehabilitation Medicine, Paris, v. 54, n. 2, p. 88-108, 2011. doi: http://dx.doi.org/10.1016/j.rehab.2011.01.001

CALLAGHAN, M.J.; MCCARTHY, C.J.; OLDHAM, J.A. The reliability of surface 
electromyography to assess quadriceps fatigue during multi joint tasks in healthy and painful knees. Journal of Electromyography and Kinesiology, Oxford, v. 19, n. 1, p.172-80, 2009. doi:

http://dx.doi.org/10.1016/j.jelekin.2007.05.004

CIFREK, M., MEDVED, V. TONKOVIC, S., OSTOJIC, S. Surface EMG based muscle fatigue evaluation in biomechanics. Clinical

Biomechanics, Huddersfield, v. 24, n. 4, p. 32740, 2009. doi:

http://dx.doi.org/10.1016/j.clinbiomech.2009.01.0 $\underline{10}$

DE LUCA, C.J. The use of surface electromyography in biomechanics. / Utilisation de I ' electromyographie de surface en biomecanique. Journal of Applied Biomechanics, Champaign, v. 13, n. 2, p. 13563, 1997. Disponível em: < http://www.bu.edu/nmrc/files/2010/04/078.pdf >. Acesso em: 5 dez 2010.

DIDERIKSEN, J.L., D. FARINA, AND R.M. ENOKA, Influence of fatigue on the simulated relation between the amplitude of the surface electromyogram and muscle force.

Philosophical Transactions of the Royal Society a-Mathematical Physical and Engineering Sciences, Londres, v. 368, n. 1920, p. 2765-81, 2010. doi:

http://dx.doi.org/10.1098/rsta.2010.0094

EBERSOLE, K.T.; O'CONNOR, K.M.; WIER, A.P. Mechanomyographic and electromyographic responses to repeated concentric muscle actions of the quadriceps femoris. Journal of Electromyography and Kinesiology, Oxford, v. 16, n. 2, p. 149-57, 2006. doi: http://dx.doi.org/10.1016/j.jelekin.2005.05.005

GONZALEZ-IZAL, M. et al. EMG spectral indices and muscle power fatigue during dynamic contractions. Journal of Electromyography and Kinesiology, Oxford, v. 20, n. 2, p. 233-240, 2010. doi:

http://dx.doi.org/10.1016/j.jelekin.2009.03.011

JACOBS, R.; VAN INGEN SCHENAU, G.J. Control of an external force in leg extensions in humans. Journal of Physiology, Cambridge, v. 457, p. 611-26, 1992.

JOHNSON, M.A.; POLGAR, J.; WEIGHTMAN, D.; APPLETON, D. Data on the distribution of fibre types in thirty-six human muscles. An autopsy study. Journal of the Neurological Sciences, New York, v. 18, n. 1, p. 111-129, 1973. doi: http://dx.doi.org/10.1016/0022$\underline{510 \times(73) 90023-3}$
KELLIS, E.; KATIS, A. Reliability of EMG powerspectrum and amplitude of the semitendinosus and biceps femoris muscles during ramp isometric contractions. Journal of Electromyography and Kinesiology, Oxford, v. 18, n. 3, p. 351-8, 2008. doi:

http://dx.doi.org/10.1016/j.jelekin.2006.12.001

KOUZAKI, M.; SHINOHARA, M.; FIKUNAGA, T. Nonuniform mechanical activity of quadriceps muscle during fatigue by repeated maximal voluntary contraction in humans. European journal of applied physiology and occupational physiology, Berlin, v. 80, n. 1, p. 9-15, 1999. doi:

http://dx.doi.org/10.1007/s004210050551

KUMAR, S. Localized muscle fatigue: review of three experiments. Revista Brasileira de

Fisioterapia, São Carlos, v. 10, p. 9-28, 2006. doi: http://dx.doi.org/10.1590/S141335552006000100003

LARIVIÈRE, C.; GAGNON, D.; GRAVEL, D.; BERTRAND-ARSENAULT, $A$. The assessment of back muscle capacity using intermittent static contractions. Part I - Validity and reliability of electromyographic indices of fatigue. Journal of Electromyography and Kinesiology, Oxford, v. 18, n. 6, p. 1006-19, 2008. doi:

http://dx.doi.org/10.1016/j.jelekin.2007.03.012

LEE, K. Y.; SHIN, K. Y.; KIM, H. S.; MUN, J. H. Estimating muscle fatigue of the biceps brachii using high to low band ratio in EMG during isotonic exercise. International Journal of Precision Engineering and Manufacturing, Seoul, v.10, n.3, Jul, p. 147-153. 2009. doi: http://dx.doi.org/10.1007/s12541-009-0060-x

LOWERY, M.; NOLAN, P.; O'MALLEY, M. Electromyogram median frequency, spectral compression and muscle fibre conduction velocity during sustained sub-maximal contraction of the brachioradialis muscle. Journal of Electromyography and Kinesiology, Oxford, v. 12, n. 2, p. 111-18, 2002. doi: http://dx.doi.org/10.1016/S1050-6411(02)00004-4

MATHUR, S.; ENG, J.J.; MACINTYRE, D.L. Reliability of surface EMG during sustained contractions of the quadriceps. Journal of Electromyography and Kinesiology, Oxford, v. 15, n. 1, p. 102-10, 2005. doi:

http://dx.doi.org/10.1016/j.jelekin.2004.06.003

OLIVEIRA, A.D.S.C.; GONÇALVES, M. EMG amplitude and frequency parameters of muscular activity: Effect of resistance training based on electromyographic fatigue threshold. Journal of Electromyography and Kinesiology, Oxford, v. 19, p. 295-303, 2009. doi: http://dx.doi.org/10.1016/i.jelekin.2007.07.008 
PINCIVERO, D.M. Knee extensor torque and quadriceps femoris EMG during perceptuallyguided isometric contractions. Journal of Electromyography and Kinesiology, Oxford, v. 13, n. 2, p.159-167, 2003. doi:

http://dx.doi.org/10.1016/S1050-6411(02)00096-2

POMPEU, F.A.M.S. Guia para estudos em biodinâmica do movimento humano. São Paulo, Ph editora, 1 edição, 2006.

PORTNEY, L.G.; WATKINS, M. P. Foundations of Clinical Research: Applications to Practice. Pearson/Prentice Hall, 2009.

ROGERS, D.R.; MACISAAC, D.T. Training a multivariable myoelectric mapping function to estimate fatigue. Journal of Electromyography and Kinesiology, Oxford, v. 20, n. 5, p. 953-60, 2010. doi:

http://dx.doi.org/10.1016/j.jelekin.2009.11.001

SANTOS, M.C.A.; SEMEGHUINI, T.A.; AZEVEDO, F.M.; COLUGNATI, D.B.; NEGRÃOFILHO, R.F.; ALVES, N. Análise da fadiga muscular em atletas e sedentários através de parâmetros de frequência do sinal eletromiográfico. Revista Brasileira de Medicina do Esporte, São Paulo, v. 14, n. 6, p. 514-17, 2008. doi: http://dx.doi.org/10.1590/S151786922008000600007

SOO, Y.; SUGI, M.; YOKOI, H.; ARAI, T.; DU, R.; OTA, J. Simultaneous measurement of force and muscle fatigue using frequency-band wavelet analysis. Conference Proceedings of the $30^{\text {th }}$ Annual International Conference of the IEEE Engineering in Medicine and Biology Society, Vancouver, p. 5045-48, 2008. doi: http://dx.doi.org/10.1109/IEMBS.2008.4650347

SOO, Y.; SUGI, M.; YOKOI, H.; ARAI, T.; NISHINO, M.; KATO, R.; NAKAMURA, T.; OTA, $J$. Estimation of handgrip force using frequencyband technique during fatiguing muscle contraction. Journal of Electromyography and Kinesiology, Oxford, v.20, n.5, p. 888-895. 2010. doi:

http://dx.doi.org/10.1016/j.jelekin.2009.08.008

YASSIERLI; NUSSBAUM, M.A. Utility of traditional and alternative EMG-based measures of fatigue during low-moderate level isometric efforts. Journal of Electromyography and Kinesiology, Oxford, v. 18, n. 1, p. 44-53, 2008. doi:

http://dx.doi.org/10.1016/j.jelekin.2006.08.003

ZWARTS, M.J., G. BLEIJENBERG, AND B.G.M. VAN ENGELEN, Clinical neurophysiology of fatigue. Clinical Neurophysiology, Amsterdam, v. 119 , n. 1 , p. $2-10,2008$. doi:

http://dx.doi.org/10.1016/j.clinph.2007.09.126
Apoio: Fundação de Amparo à Pesquisa do Estado de São Paulo (processo: 2008/11639-4).

\section{Endereço:}

Fábio Mícolis de Azevedo

UNESP - Departamento de Fisioterapia

Rua Roberto Simonsen, 305 Caixa-postal: 967

Presidente Prudente SP Brasil 19060-900

Telefone: (24) 3229-5365 - ramal: 5416

e-mail: micolis@fct.unesp.br

Recebido em: 1 de fevereiro de 2011.

Aceito em: 21 de maio de 2012.

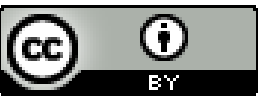

Motriz. Revista de Educação Física. UNESP, Rio Claro, SP, Brasil - elSSN: 1980-6574 - está licenciada sob Creative Commons - Atribuição 3.0 